\title{
Heat rectification with a minimal model of two harmonic oscillators
}

\author{
M. A. Simón $\odot,{ }^{1, *}$ A. Alaña $\odot,{ }^{1}$ M. Pons $\odot,{ }^{2}$ A. Ruiz-García $\odot,{ }^{3,4}$ and J. G. Muga $\odot^{1, \dagger}$ \\ ${ }^{1}$ Departamento de Química-Física, Universidad del País Vasco, UPV/EHU, Bilbao, Spain \\ ${ }^{2}$ Departamento de Física Aplicada I, Universidad del País Vasco, UPV/EHU, Bilbao, Spain \\ ${ }^{3}$ Departamento de Física, Universidad de La Laguna, La Laguna 38203, Spain \\ ${ }^{4}$ Instituto Universitario de Estudios Avanzados (IUdEA), Universidad de La Laguna, La Laguna 38203, Spain
}

(Received 20 October 2020; revised 22 December 2020; accepted 10 January 2021; published 27 January 2021)

\begin{abstract}
We study heat rectification in a minimalistic model composed of two unequal atoms subjected to linear forces and in contact with effective Langevin baths induced by Doppler lasers. Analytic expressions of the heat currents in the steady state are spelled out. Asymmetric heat transport is found in this linear system if both the bath temperatures and the temperature-dependent bath-system couplings are exchanged. The model can be realized with two ions in either common or individual traps. This physical setting allows for a natural temperature dependence of the coupling to the baths. We also explore the parameter space of the model to optimize asymmetric heat current and find conditions for maximal rectification. High rectification corresponds to a good match of the power spectra of the ions for forward temperature bias and mismatch for reverse bias, which may be understood by the behavior of dissipative normal modes.
\end{abstract}

DOI: 10.1103/PhysRevE.103.012134

\section{INTRODUCTION}

Heat rectification is the physical phenomenon, analogous to electrical current rectification in diodes, in which heat current through a device or medium (the thermal diode or rectifier) is not symmetric with respect to the exchange of the bath temperatures at the boundaries. It was first observed in 1936 by Starr in a junction between copper and cuprous oxide [1]. The theoretical work started much later using as rectifiers simple anharmonic chain models with different segments $[2,3]$. These papers sparked much research that continues to this day. The field remains very active, driven by potential applications in fundamental science and technology for thermal management and signal processing and also because none of the proposals so far appears to be efficient enough for practical purposes, i.e., highly conducting in one direction and insulating in the other one. The studies have also branched into different subfields and systems (e.g., quantum or classical [4], and for macroscopic, mesoscopic, or microscopic devices) that need specific treatments. A full account of the developments and results is out of the scope of this Introduction, but several good reviews are available for a broad perspective [4-7]. We merely mention in passing important progress on nanostructures [6,7], macroscopic devices [5], or time-dependent drivings [6,8]. Instead we focus on some aspects more closely connected to the present work that help to motivate it and put it in context. The rectification in the first models was explained by the different temperature dependencies of the phonon bands (power spectra) of the segments [2,3]. A match or mismatch

\footnotetext{
*miguelangel.simon@ehu.eus

†jg.muga@ehu.es
}

of the spectra of neighboring parts implies corresponding good or bad conduction so the sign of the temperature bias may affect the conduction and lead to rectification when the spectra of the parts are affected differently by the bias reversal. Interaction potential anharmonicities (i.e., nonlinear forces) imply dependencies of the spectra on temperature and thus have been regarded recurrently as an essential element for rectification [6,9-15]. However, Pereira pointed out [16] that anharmonicity is not a necessary condition for rectification. Rectification also occurs in simple (minimalistic) harmonic models that incorporate some structural asymmetry and temperature dependence of the model parameters. This dependence may indeed result from an underlying, more intricate anharmonic system by linearization of the stochastic dynamics $[4,16]$, or it may have a different origin [17]. In general, minimalistic models, harmonic or otherwise, provide insight and serve to guide further work towards effective rectifiers.

To look for higher rectification factors, the use of graded materials [18] and long-range interactions (LRIs) was put forward $[19,20]$. It was noted recently that LRIs naturally occur as a result of the Coulomb interaction in a chain of cold ions in Paul traps [17], and that this system may serve to bridge the gap between simple models and experimental realizations. In Ref. [17] the gradation was incorporated by ramping the frequencies of individual traps. Moreover, the linear regime (when the potentials are well approximated harmonically) is realistic for trapped ions, and shows rectification because of the temperature dependence of the coupling to the effective bath induced by Doppler cooling lasers. Trapped ions constitute a well-developed and tested architecture for fundamental research, quantum information processing, and quantum technologies such as detectors or metrology. This architecture is in principle scalable in driven ion circuits (see, 
e.g., Ref. [21]). Controllable heat rectification in this context would be a useful asset for energy management.

In this article we put forward, along this line of trapped-ion physics, an even simpler, minimalistic model for rectification implemented by two neighboring atoms of different mass interacting harmonically and in contact with thermal baths with temperature-dependent couplings (LRI does not play any role in the two-ion configuration but it would affect the dynamics of longer chains). The model admits a natural realization in terms of two trapped ions subjected to Doppler cooling lasers, which provide the necessary temperature dependence of the coupling parameters. Apart from the possibility of a physical realization, another interesting feature is the analytical treatment, which facilitates greatly the exploration in parameter space to identify regimes of maximal rectification. The explicit solution of the stationary regime also provides tools for a better understanding of the physics and enhanced control. For example, the match or mismatch of the spectra of the two masses for forward and reverse bias configurations, which will be made evident for the parameters with maximal rectification, may be analyzed in terms of dissipative normal modes characterized by complex eigenvalues.

The model may be compared and related to other simple models. The localization of the structural asymmetry in a small spatial region, by a "defect," "impurity," or asymmetrical molecule, has been proposed, e.g., in several anharmonic models [13,22,23]. Segal and Nitzan proposed models with some similarities to ours $[12,13]$, specifically an anharmonic chain with different couplings to both baths. They also worked out quantum models [12,13] in terms of an $N$-level system asymmetrically coupled to the baths. Both types of models have a "harmonic limit," which in the chain is reached by making the potentials harmonic, and in the quantum one by taking $N$ to infinity assuming equispaced levels. The asymmetrical couplings, however, were not interchanged when reversing the temperature bias (in these models that interchange would have suppressed the asymmetry because the forward bias configuration becomes a mirror image of the reverse bias one), so that the harmonic limit did not give any rectification.

The article is organized as follows: In Sec. II we describe the physical model and its dynamical equations. In Sec. III we introduce the covariance matrix and derive the equation that it satisfies in the steady state. In Sec. IV we solve the covariance matrix equation and find analytical expressions for the steadystate temperatures of the masses and heat currents. In Sec. V we relate the parameters of the model to those of Doppler cooled trapped ions. In Sec. VI we look for configurations with high rectification. We also study the power spectra of the oscillators, which confirm the match or mismatch pattern for rectification. Finally, in Sec. VII we summarize the results and present the conclusions.

\section{PHYSICAL MODEL}

The physical model consists of two masses $m_{1}$ and $m_{2}$ coupled to each other by a harmonic interaction with spring constant $k$ and natural length $x_{e}$. The masses $m_{1}$ and $m_{2}$ are confined by harmonic potentials centered at $x_{L}, x_{R}$ with spring constants $k_{L}, k_{R}$, respectively (see Fig. 1). The Hamiltonian

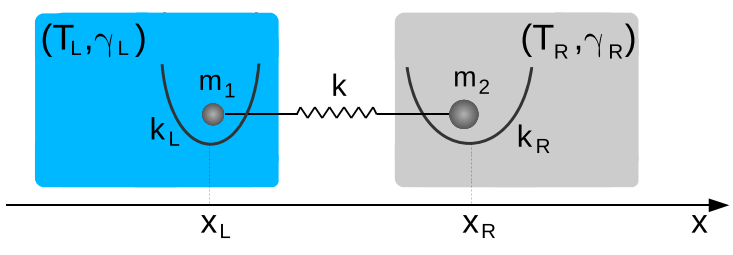

FIG. 1. Diagram of the model described in Sec. II. Two masses are coupled to each other through a spring constant $k$. Each mass is harmonically trapped and connected to a bath characterized by its temperature $T_{i}$ and its friction coefficient $\gamma_{i}$.

describing this model is

$$
H=\frac{p_{1}^{2}}{2 m_{1}}+\frac{p_{2}^{2}}{2 m_{2}}+V\left(x_{1}, x_{2}\right),
$$

with $\quad V\left(x_{1}, x_{2}\right)=\frac{k}{2}\left(x_{1}-x_{2}-x_{e}\right)^{2}+\frac{k_{L}}{2}\left(x_{1}-x_{L}\right)^{2}+$ $\frac{k_{R}}{2}\left(x_{2}-x_{R}\right)^{2}$, where $\left\{x_{i}, p_{i}\right\}_{i=1,2}$ are the position and momentum of each mass. Switching from the original coordinates $x_{i}$ to displacements with respect to the equilibrium positions of the system, $q_{i}=x_{i}-x_{i}^{e q}$, where $x_{i}^{e q}$ are the solutions to $\partial_{x_{i}} V\left(x_{1}, x_{2}\right)=0$, the Hamiltonian can be written as

$$
\begin{aligned}
H= & \frac{p_{1}^{2}}{2 m_{1}}+\frac{p_{2}^{2}}{2 m_{2}}+\frac{k+k_{L}}{2} q_{1}^{2} \\
& +\frac{k+k_{R}}{2} q_{2}^{2}-k q_{1} q_{2}+V\left(x_{1}^{e q}, x_{2}^{e q}\right) .
\end{aligned}
$$

Dropping the constant term, this has the form of the Hamiltonian of a system around a stable equilibrium point,

$$
H=\frac{1}{2} \vec{p}^{\top} \mathbb{M}^{-1} \vec{p}+\frac{1}{2} \vec{q}^{\top} \mathbb{K} \vec{q}
$$

where $\vec{q}=\left(q_{1}, q_{2}\right)^{\top}, \vec{p}=\left(p_{1}, p_{2}\right)^{\top}, \mathbb{M}=\operatorname{diag}\left(m_{1}, m_{2}\right)$ is the mass matrix of the system, and $\mathbb{K}$ is the Hessian matrix of the potential at the equilibrium point, i.e., $\mathbb{K}_{i j}=$ $\left.\partial_{x_{i}, x_{j}}^{2} V(\vec{x})\right|_{\vec{x}=\vec{x}^{e q}}$. In this model $\mathbb{K}_{11}=k+k_{L}, \mathbb{K}_{22}=k+$ $k_{R}$, and $\mathbb{K}_{12}=\mathbb{K}_{21}=-k$. We see later that the generic form of Eq. (3) can be adapted to different physical settings, in particular to two ions in individual traps, or to two ions in a common trap.

The masses are in contact with Langevin baths, which will be denoted as $L$ (for left) and $R$ (for right), at temperatures $T_{L}$ and $T_{R}$ for the masses $m_{1}$ and $m_{2}$, respectively (see Fig. 1). The equations of motion of the system, taking into account the Hamiltonian and the Langevin baths, are

$$
\begin{aligned}
& \dot{q}_{1}=\frac{p_{1}}{m_{1}}, \quad \dot{q}_{2}=\frac{p_{2}}{m_{2}}, \\
& \dot{p}_{1}=-\left(k+k_{L}\right) q_{1}+k q_{2}-\frac{\gamma_{L}}{m_{1}} p_{1}+\xi_{L}(t), \\
& \dot{p}_{2}=-\left(k+k_{R}\right) q_{2}+k q_{1}-\frac{\gamma_{R}}{m_{2}} p_{2}+\xi_{R}(t),
\end{aligned}
$$

where $\gamma_{L}, \gamma_{R}$ are the friction coefficients of the baths and $\xi_{L}(t), \xi_{R}(t)$ are Gaussian white-noise-like forces. The Gaussian forces have zero mean over noise realizations $\left(\left\langle\xi_{L}(t)\right\rangle=\right.$ $\left.\left\langle\xi_{R}(t)\right\rangle=0\right)$ and satisfy the correlations $\left\langle\xi_{L}(t) \xi_{R}\left(t^{\prime}\right)\right\rangle=0$, $\left\langle\xi_{L}(t) \xi_{L}\left(t^{\prime}\right)\right\rangle=2 D_{L} \delta\left(t-t^{\prime}\right)$, and $\left\langle\xi_{R}(t) \xi_{R}\left(t^{\prime}\right)\right\rangle=2 D_{R} \delta\left(t-t^{\prime}\right)$. 
$D_{L}$ and $D_{R}$ are the diffusion coefficients, which satisfy the fluctuation-dissipation theorem, $D_{L}=\gamma_{L} k_{B} T_{L}, D_{R}=\gamma_{R} k_{B} T_{R}$, where $k_{B}$ is the Boltzmann constant.

It is useful to define the phase-space vector $\vec{r}(t)=$ $\left(\vec{q}, \mathbb{M}^{-1} \vec{p}\right)^{\top}$ (note that $\vec{v}=\mathbb{M}^{-1} \vec{p}$ is just the velocity vector). The equations of motion are

$$
\dot{\vec{r}}(t)=\mathbb{A} \vec{r}(t)+\mathbb{L} \vec{\xi}(t),
$$

with

$$
\begin{aligned}
\mathbb{A} & =\left(\begin{array}{cc}
\mathbb{D}_{2 \times 2} & \mathbb{1}_{2 \times 2} \\
-\mathbb{M}^{-1} \mathbb{K} & -\mathbb{M}^{-1} \mathbb{\Gamma}
\end{array}\right), \\
\mathbb{L} & =\left(\begin{array}{c}
\mathbb{D}_{2 \times 2} \\
\mathbb{M}^{-1}
\end{array}\right),
\end{aligned}
$$

and $\vec{\xi}(t)=\left(\xi_{L}(t), \xi_{R}(t)\right)^{\top}, \mathbb{\Gamma}=\operatorname{diag}\left(\gamma_{L}, \gamma_{R}\right) . \mathbb{D}_{n \times n}$ and $\mathbb{1}_{n \times n}$ are the $n$-dimensional squared zero matrix and identity matrix, respectively. With the vector notation the correlation of the white-noise forces can be written as

$$
\left\langle\vec{\xi}(t) \vec{\xi}\left(t^{\prime}\right)^{\top}\right\rangle=2 \mathbb{D} \delta\left(t-t^{\prime}\right)
$$

where $\mathbb{D}=\operatorname{diag}\left(D_{L}, D_{R}\right)$.

\section{COVARIANCE MATRIX IN THE STEADY STATE}

We define the covariance matrix of the system as

$$
\mathbb{C}(t)=\left\langle\vec{r}(t) \vec{r}(t)^{\top}\right\rangle .
$$

This matrix is important because the heat transport properties can be extracted from it. In particular, the kinetic temperatures of the masses, $T_{1}(t)$ and $T_{2}(t)$, are

$$
\begin{aligned}
& T_{1}(t)=\frac{\left\langle p_{1}^{2}(t)\right\rangle}{m_{1} k_{B}}=\frac{m_{1} C_{3,3}(t)}{k_{B}}, \\
& T_{2}(t)=\frac{\left\langle p_{2}^{2}(t)\right\rangle}{m_{2} k_{B}}=\frac{m_{2} C_{4,4}(t)}{k_{B}} .
\end{aligned}
$$

One approach to find the covariance matrix is to solve Eq. (5). However, this requires solving the equations explicitly or simulating them numerically many times to find the covariance matrix for the ensemble of simulated stochastic trajectories. Instead, we proceed by looking for an ordinary differential equation that gives the evolution of the covariance matrix as described in Refs. [24-26]. Differentiating $\mathbb{C}(t)$ with respect to time and using Eq. (5) we get

$$
\begin{aligned}
\frac{d}{d t} \mathbb{C}(t)= & \mathbb{A} \mathbb{C}(t)+\mathbb{C}(t) \mathbb{A}^{\top} \\
& +\mathbb{L}\left\langle\vec{\xi}(t) \vec{r}(t)^{\top}\right\rangle+\left\langle\vec{r}(t) \vec{\xi}(t)^{\top}\right\rangle \mathbb{L}^{\top} .
\end{aligned}
$$

The solution of Eq. (10) allows us to find the local temperatures of the masses as a function of the bath temperatures [Eq. (9)] at all times. In particular, we are interested in the covariance matrix in the steady state, i.e., for $t \rightarrow \infty$.

In the steady state, the covariance matrix is constant $\left(\frac{d}{d t} \mathbb{C}(t)=0\right)$; therefore, it satisfies

$$
\mathbb{A} \mathbb{C}^{\text {s.s. }}+\mathbb{C}^{\text {s.s. }} \mathbb{A}^{\top}=-\mathbb{L}\left\langle\vec{\xi} \vec{r}^{\top}\right\rangle^{\text {s.s. }}-\left\langle\vec{r} \vec{\xi}^{\top}\right\rangle^{\text {s.s. }} \mathbb{L}^{\top},
$$

with $\{\cdot\}^{\text {s.s. }} \equiv \lim _{t \rightarrow \infty}\{\cdot\}(t)$. Equation (11) is an algebraic equation whose solution is the steady-state covariance matrix $\mathbb{C}^{\text {s.s. }}$. However, the two terms $\left\langle\vec{\xi} \vec{r}^{\mathrm{T}}\right\rangle^{\text {s.s. }}$ and $\left\langle\vec{r} \vec{\xi}^{\mathrm{T}}\right\rangle^{\text {s.s. }}$ need to be calculated before working out the solution. Using Novikov's theorem and the $\delta$ correlation of the noises, we find the $i j$ th component of $\left\langle\vec{\xi}(t) \vec{r}(t)^{\top}\right\rangle$ without solving Eq. (5),

$$
\begin{aligned}
\left\langle\xi_{i}(t) r_{j}(t)\right\rangle & =\sum_{k=1}^{2} \int_{0}^{t} d \tau\left\langle\xi_{i}(t) \xi_{k}(\tau)\right\rangle\left\langle\frac{\delta r_{j}(t)}{\delta \xi_{k}(\tau)}\right\rangle \\
& =\sum_{k=1}^{2} \mathbb{D}_{i k} \lim _{\tau \rightarrow t^{-}}\left\langle\frac{\delta r_{j}(t)}{\delta \xi_{k}(\tau)}\right\rangle,
\end{aligned}
$$

where $\lim _{\tau \rightarrow t^{-}}$is the limit when $\tau$ goes to $t$ from below. Evaluation of the functional derivative $\delta r_{j}(t) / \delta \xi_{k}(\tau)$ for the $\tau \rightarrow t^{-}$limit gives

$$
\left\langle\vec{\xi}(t) \vec{r}(t)^{\top}\right\rangle=\mathbb{D}^{\top} .
$$

Now, the algebraic equation that gives the steady-state covariance matrix becomes

$$
\mathbb{A} \mathbb{C}^{\text {s.s. }}+\mathbb{C}^{\text {s.s. }} \mathbb{A}^{\top}=-\mathbb{B},
$$

with $\mathbb{B}=2 \mathbb{L D L} L^{\top}$. By definition, the covariance matrix is symmetric, but there are also additional restrictions imposed by the equations of motion and the steady-state condition, which reduce the dimensionality of the problem of solving Eq. (14) [17]. Since $d\left\langle q_{i} q_{j}\right\rangle / d t=0$ in the steady state, we have

$$
\begin{aligned}
\left\langle p_{1} q_{1}\right\rangle^{\text {s.s. }} & =\left\langle p_{2} q_{2}\right\rangle^{\text {s.s. }}=0, \\
\frac{\left\langle p_{1} q_{2}\right\rangle^{\text {s.s. }}}{m_{1}} & =-\frac{\left\langle q_{1} p_{2}\right\rangle^{\text {s.s. }}}{m_{2}} .
\end{aligned}
$$

Taking Eqs. (15) into account, the steady-state covariance matrix takes the form

$$
\mathbb{C}^{\text {s.s. }}=\left(\begin{array}{cccc}
\left\langle q_{1}^{2}\right\rangle^{\text {s.s. }} & \left\langle q_{1} q_{2}\right\rangle^{\text {s.s. }} & 0 & \frac{\left\langle p_{2} q_{1}\right\rangle^{\text {s.s. }}}{m_{2}} \\
\left\langle q_{1} q_{2}\right\rangle^{\text {s.s. }} & \left\langle q_{2}^{2}\right\rangle^{\text {s.s. }} & -\frac{\left\langle p_{2} q_{1}\right\rangle^{\text {s.s. }}}{m_{2}} & 0 \\
0 & -\frac{\left\langle p_{2} q_{1}\right\rangle^{\text {s.s. }}}{m_{2}} & \frac{\left\langle p_{1}^{2}\right\rangle^{\text {s.s. }}}{m_{1}^{2}} & \frac{\left\langle p_{1} p_{2}\right\rangle^{\text {s.s. }}}{m_{1} m_{2}} \\
\frac{\left\langle p_{2} q_{1}\right\rangle^{\text {s.s. }}}{m_{2}} & 0 & \frac{\left\langle p_{1} p_{2}\right\rangle^{\text {s.s. }}}{m_{1} m_{2}} & \frac{\left\langle p_{2}^{2}\right\rangle^{\text {s.s. }}}{m_{2}^{2}}
\end{array}\right) .
$$

The explicit set of equations for the components of $\mathbb{C}^{\text {s.s. }}$ can be found in Appendix A.

\section{SOLUTIONS}

In this section we use the solution to Eq. (14) to write down the temperatures and currents in the steady state. We use MATHEMATICA to find analytic expressions for the temperatures,

$$
\begin{aligned}
& T_{1}=\frac{T_{L} \mathcal{P}_{1, L}(k)+T_{R} \mathcal{P}_{1, R}(k)}{\mathcal{D}(k)}, \\
& T_{2}=\frac{T_{L} \mathcal{P}_{2, L}(k)+T_{R} \mathcal{P}_{2, R}(k)}{\mathcal{D}(k)},
\end{aligned}
$$


where $\mathcal{D}(k)=\sum_{n=0}^{2} \mathcal{D}_{n} k^{n}$ and $\mathcal{P}_{i,(L / R)}(k)=\sum_{n=0}^{2} a_{i, n,(L / R)} k^{n}$ are polynomials in the coupling constant $k$ with coefficients

$$
\begin{aligned}
\mathcal{D}_{0} & =a_{1,0, L}=a_{2,0, R} \\
& =\gamma_{L} \gamma_{R}\left[h^{(1)}\left(\gamma_{L} k_{R}+\gamma_{R} k_{L}\right)+\left(m_{1} k_{R}-m_{2} k_{L}\right)^{2}\right], \\
\mathcal{D}_{1} & =a_{1,1, L}=a_{2,1, R} \\
& =\gamma_{L} \gamma_{R}\left[h^{(0)} h^{(1)}+2\left(m_{1}-m_{2}\right)\left(m_{1} k_{R}-m_{2} k_{L}\right)\right], \\
\mathcal{D}_{2} & =h^{(0)} h^{(2)}, \\
a_{1,2, L} & =\gamma_{L}\left(m_{2} h^{(1)}+\gamma_{R}\left(m_{1}-m_{2}\right)^{2}\right), \\
a_{1,2, R} & =h^{(1)} m_{1} \gamma_{R}, \\
a_{2,2, L} & =h^{(1)} m_{2} \gamma_{L}, \\
a_{2,2, R} & =\gamma_{R}\left(m_{1} h^{(1)}+\gamma_{L}\left(m_{1}-m_{2}\right)^{2}\right), \\
a_{1,0, R} & =a_{1,1, R}=a_{2,0, L}=a_{2,1, L}=0,
\end{aligned}
$$

where

$$
h^{(n)} \equiv \gamma_{R} m_{1}^{n}+\gamma_{L} m_{2}^{n}
$$

The currents from the baths to the masses [17] are

$$
J_{L}=k_{B} \frac{\gamma_{L}}{m_{1}}\left(T_{L}-T_{1}\right), \quad J_{R}=k_{B} \frac{\gamma_{R}}{m_{2}}\left(T_{R}-T_{2}\right),
$$

with $T_{i}$ given by Eq. (17). Since, in the steady state, $J_{L}=$ $-J_{R}$, we will use the shorthand notation $J \equiv J_{L}$. Substituting Eq. (17) into Eq. (20) we get for the heat current

$$
J=\kappa\left(T_{L}-T_{R}\right),
$$

where $\kappa=k_{B} k^{2} \gamma_{L} \gamma_{R} h^{(1)} / \mathcal{D}(k)$ acts as an effective thermal conductance.

\section{RELATION OF THE MODEL TO A TRAPPED-ION SETUP}

In this section we discuss the realization of the model with a pair of trapped ions. We consider two different setups: two ions in a collective trap, and two ions in individual traps. Later in Sec. VI we focus on two ions in individual traps to illustrate the analysis of rectification.

In both setups we assume strong confinement in the radial direction, making the effective dynamics one dimensional. We will also assume that the confinement in the axial direction is purely electrostatic, which makes the effective spring constant independent of the mass of the ions [27]. Additionally, we will relate the temperatures and friction coefficients of the Langevin baths to those corresponding to Doppler cooling.

\section{A. Collective trap}

Consider two ions of unit charge with masses $m_{1}$ and $m_{2}$ trapped in a collective trap. Assuming strong radial confinement and purely electrostatic axial confinement, both ions feel the same harmonic oscillator potential with trapping constant $k_{\text {trap }}$ [27]. The potential describing the system is

$$
V_{\text {collective }}=\frac{1}{2} k_{\text {trap }}\left(x_{1}^{2}+x_{2}^{2}\right)+\frac{\mathcal{C}}{x_{2}-x_{1}},
$$

with $\mathcal{C}=Q^{2} /\left(4 \pi \varepsilon_{0}\right)$. The equilibrium positions for this potential are

$$
x_{2}^{e q}=-x_{1}^{e q}=\left(\frac{1}{2}\right)^{2 / 3}\left(\frac{Q^{2}}{4 \pi \varepsilon_{0} k_{\text {trap }}}\right)^{1 / 3} .
$$

Assuming small oscillations of the ions around the equilibrium positions, the Hessian matrix of the system is

$$
\begin{aligned}
& \mathbb{K}_{1,2}=-\frac{Q^{2}}{2 \pi \varepsilon_{0}} \frac{1}{\left(x_{2}^{e q}-x_{1}^{e q}\right)^{3}}=-k_{\text {trap }}, \\
& \mathbb{K}_{1,1}=k_{\text {trap }}+\frac{Q^{2}}{2 \pi \varepsilon_{0}} \frac{1}{\left(x_{2}^{e q}-x_{1}^{e q}\right)^{3}}=2 k_{\text {trap }}, \\
& \mathbb{K}_{2,2}=k_{\text {trap }}+\frac{Q^{2}}{2 \pi \varepsilon_{0}} \frac{1}{\left(x_{2}^{e q}-x_{1}^{e q}\right)^{3}}=2 k_{\text {trap }} .
\end{aligned}
$$

Using Eqs. (24) we can relate the parameters of this physical setup to those of the model described in Sec. II,

$$
k_{L}=k_{R}=k=k_{\text {trap }} .
$$

\section{B. Individual on-site traps}

We can make the same assumptions for the axial confinement as in the previous section but now each of the ions is in an individual trap with spring constants $k_{\text {trap }, L}$ and $k_{\operatorname{trap}, R}$, respectively. The potential of the system is

$$
\begin{aligned}
V_{\text {individual }}= & \frac{1}{2} k_{\text {trap }, L}\left(x_{1}-x_{L}\right)^{2}+\frac{1}{2} k_{\text {trap }, R}\left(x_{2}-x_{R}\right)^{2} \\
& +\frac{\mathcal{C}}{x_{2}-x_{1}},
\end{aligned}
$$

where $x_{L}$ and $x_{R}$ are the center positions of the on-site traps. The elements of the Hessian matrix in the equilibrium position are

$$
\begin{aligned}
& \mathbb{K}_{1,2}=-\frac{Q^{2}}{2 \pi \varepsilon_{0}} \frac{1}{\left(x_{2}^{e q}-x_{1}^{e q}\right)^{3}}, \\
& \mathbb{K}_{1,1}=k_{\text {trap }, L}+\frac{Q^{2}}{2 \pi \varepsilon_{0}} \frac{1}{\left(x_{2}^{e q}-x_{1}^{e q}\right)^{3}}, \\
& \mathbb{K}_{2,2}=k_{\text {trap }, R}+\frac{Q^{2}}{2 \pi \varepsilon_{0}} \frac{1}{\left(x_{2}^{e q}-x_{1}^{e q}\right)^{3}} .
\end{aligned}
$$

Comparing the parameters in Eq. (27) with those in the model described in Sec. II, we identify

$$
\begin{aligned}
k_{L} & =k_{\text {trap }, L}, \\
k_{R} & =k_{\text {trap }, R}, \\
k & =\frac{Q^{2}}{2 \pi \varepsilon_{0}} \frac{1}{\left(x_{2}^{e q}-x_{1}^{e q}\right)^{3}} .
\end{aligned}
$$

In this case, the analytic expressions for the equilibrium positions are more complicated. We get for the distance between the equilibrium positions of the ions 


$$
\left(x_{2}-x_{1}\right)^{(e q)}=\frac{1}{3} \Delta x_{L R}-\frac{1}{6}\left[\frac{2^{2 / 3} \zeta}{k_{\text {trap }, L} k_{\text {trap }, R}\left(k_{\text {trap }, L}+k_{\text {trap }, R}\right)}+\frac{2^{4 / 3} k_{\text {trap }, L} k_{\text {trap }, R}\left(k_{\text {trap }, L}+k_{\text {trap }, R}\right)\left(x_{R}-x_{L}\right)^{2}}{\zeta}\right],
$$

where $\Delta x_{L R}=\left(x_{R}-x_{L}\right)$ and $\zeta=(Y-\eta)^{(1 / 3)}$, with

$$
\begin{aligned}
Y & =3 \sqrt{3}\left\{\mathcal{C} k_{\text {trap }, L}^{4} k_{\text {trap }, R}^{4}\left(k_{\text {trap }, L}+k_{\text {trap }, R}\right)^{7}\left[4 k_{\text {trap }, L} k_{\text {trap }, R} \Delta x_{L R}^{3}+27 \mathcal{C}\left(k_{\text {trap }, L}+k_{\text {trap }, R}\right)\right]\right\}^{1 / 2}, \\
\eta & =k_{\text {trap }, L}^{2} k_{\text {trap }, R}^{2}\left(k_{\text {trap }, L}+k_{\text {trap }, R}\right)^{3}\left[2 k_{\text {trap }, L} k_{\text {trap }, R} \Delta x_{L R}^{3}+27 \mathcal{C}\left(k_{\text {trap }, L}+k_{\text {trap }, R}\right)\right] .
\end{aligned}
$$

In this setup, the coupling between the ions $k$ can be controlled by changing the distance between the on-site traps.

\section{Optical molasses and Langevin baths}

Trapped ions may be cooled down by counterpropagating lasers which are red detuned with respect to an internal atomic transition of the ions. This technique is known as Doppler cooling or optical molasses [28-31]. The off-resonant absorption of laser photons by the ions exerts a dampinglike force that slows them down. The spontaneous emission of the ions produces heating due to the random recoil generated by the emitted photons. The friction and recoil force balance, so eventually the ion thermalizes to a finite temperature. Thus the effect of the lasers on the ion is equivalent to a Langevin bath with temperature $T_{\text {molass }}$ and friction coefficient $\gamma_{\text {molass }}$. The temperature and friction coefficients are controlled with the laser intensity $I$ and frequency detuning $\delta$ with respect to the selected internal atomic transition by the expressions $[29,31,32]$

$$
\begin{aligned}
\gamma_{\text {molass }}(I, \delta) & =-4 \hbar\left(\frac{\delta+\omega_{0}}{c}\right)^{2}\left(\frac{I}{I_{0}}\right) \frac{2 \delta / \Gamma}{\left[1+(2 \delta / \Gamma)^{2}\right]^{2}}, \\
T_{\text {molass }}(\delta) & =-\frac{\hbar \Gamma}{4 k_{B}} \frac{1+(2 \delta / \Gamma)^{2}}{(2 \delta / \Gamma)},
\end{aligned}
$$

where $\omega_{0}$ is the frequency of the internal atomic transition, $\Gamma$ is the natural width (decay rate) of the excited state, and $I_{0}$ is the saturation intensity. For fixed $\Gamma$ and $I, \gamma_{\text {molass }}$ depends on $\delta$ and, thus, indirectly on the temperature $T_{\text {molass }}$.

\section{LOOKING FOR RECTIFICATION}

There is rectification if the flux $J$ for the forward temperature bias is different from the flux $\tilde{J}$ for reverse bias with the baths exchanged. To measure rectification, we will use the rectification coefficient $0 \leqslant R \leqslant 1$ defined as

$$
R=\frac{|| J|-| \tilde{J}||}{\max (|J|,|\tilde{J}|)} .
$$

The important point here is to define what is meant by $e x$ changing the baths. We consider that a bath is characterized not only by its temperature $T$ but also by its coupling to the system by means of the friction coefficient $\gamma$, so exchanging the baths is achieved by exchanging both the temperatures and the friction coefficients, as summarized in Table I. For generic models this choice is a matter of definition, but for trapped ions it is a natural way to proceed.
When implementing temperatures and friction coefficients by lasers according to Eqs. (31), the exchange operation is straightforward when the two ions are either of the same species or isotopes of each other, since the only required action to exchange temperatures is to exchange the detunings without modifying the intensities. The detuning exchange in turn automatically exchanges the friction coefficients. However, for two different species, which involve two different atomic transitions, the laser wavelengths and the decay rates $\Gamma$ depend on the species. Then, exchanging the temperatures by modifying the detunings, keeping the laser intensities constant, does not necessarily imply an exchange of the friction coefficients. Nevertheless, it is possible to adjust the laser intensities so that the friction coefficients get exchanged and that is the assumption hereafter. In terms of the analysis of rectification in Ref. [16], we are adding a temperature-dependent feature to the system; namely, the friction coefficients depend on the bath temperature and are exchanged as the baths are reversed.

\section{A. Parametric exploration}

We have explored thoroughly the space formed by the parameters of the model, $m_{1}, m_{2}, k, k_{L}, k_{R}, \gamma_{L}$, and $\gamma_{R}$, to find and maximize asymmetric heat transport. We have fixed the values of some of the parameters to realistic ones while varying the rest. Unless stated otherwise the masses are $m_{1}=$ 24.305 a.u. and $m_{2}=40.078$ a.u., which correspond to $\mathrm{Mg}$ and $\mathrm{Ca}$, whose ions are broadly used in trapped-ion physics. According to Eq. (21) and the corresponding expression for $\tilde{J}$ with the substitutions in Table I, rectification does not formally depend on the bath temperatures in this model for given friction coefficients. Of course the friction coefficients depend on the temperature indirectly, but also on laser intensities [see Eqs. (31)], so in the parametric space $m_{1}, m_{2}, k, k_{L}, k_{R}, \gamma_{L}$, and $\gamma_{R}$ there is no need to specify the bath temperatures to analyze the rectification in the following. The bath temperatures will be needed though to calculate the power spectra, and they play an implicit role in the central assumption that their exchange implies an exchange of friction coefficients.

TABLE I. Definition of forward and reversed (exchanged) bath configurations.

\begin{tabular}{lcc}
\hline \hline & Forward & Reversed \\
\hline Bath friction & $\gamma_{L}, \gamma_{R}$ & $\tilde{\gamma}_{L}=\gamma_{R}, \tilde{\gamma}_{R}=\gamma_{L}$ \\
Bath temperature & $T_{L}, T_{R}$ & $\tilde{T}_{L}=T_{R}, \tilde{T}_{R}=T_{L}$ \\
\hline \hline
\end{tabular}




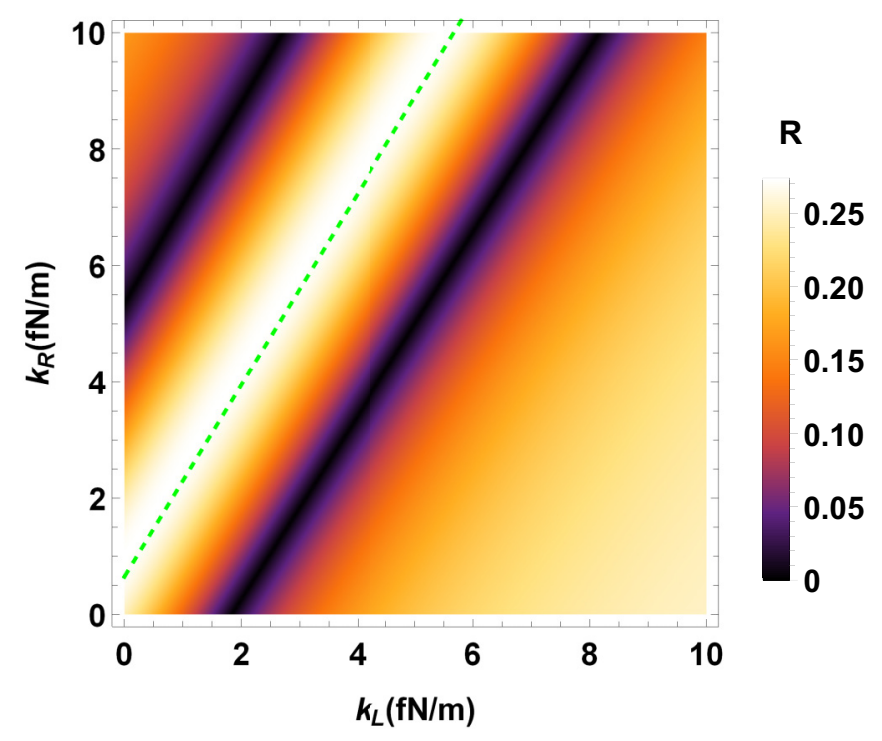

FIG. 2. Rectification, $R$, in the $k_{L} k_{R}$ plane for $k=1.17 \mathrm{fN} / \mathrm{m}$, $\gamma_{L}=6.75 \times 10^{-22} \mathrm{~kg} / \mathrm{s}$, and $\gamma_{R}=4.64 \gamma_{L}, m_{1}=24.305$ a.u., and $m_{2}=40.078$ a.u. The dashed line represents Eq. (33).

Figure 2 depicts the values of the rectification after sweeping the $k_{L} k_{R}$ plane for fixed values of $k, \gamma_{L}$, and $\gamma_{R}$. There is a ridge in the $k_{L} k_{R}$ plane for which the rectification is maximal. $\partial_{k_{L}} R=0$ may be solved explicitly but the solution is too long to be displayed here. In a weak dissipation regime $\left(\gamma_{L} / m_{1} \ll \sqrt{k / m_{1}}, \gamma_{R} / m_{2} \ll \sqrt{k / m_{2}}\right)$, a Taylor series around $\left(\gamma_{L}, \gamma_{R}\right)=(0,0)$ gives in zeroth order a straight line for the ridge,

$$
\frac{k+k_{R}}{m_{2}}=\frac{k+k_{L}}{m_{1}} .
$$

Equation (33) implies the resonance condition $\omega_{L}=\omega_{R}$ for the effective oscillation frequencies $\omega_{L}=\sqrt{\left(k+k_{L}\right) / m_{1}}$ and $\omega_{R}=\sqrt{\left(k+k_{R}\right) / m_{2}}$ [see Eq. (2)]. The lowest order correction to Eq. (33) implies a small shift of the line, keeping the same slope,

$$
\frac{k+k_{R}}{m_{2}}=\frac{k+k_{L}}{m_{1}}+\frac{\left(m_{2} \gamma_{L}+m_{1} \gamma_{R}\right)\left(m_{1} \gamma_{L}+m_{2} \gamma_{R}\right)}{2 m_{1} m_{2}\left(m_{2}^{2}-m_{1}^{2}\right)} .
$$

In a trapped-ion context, condition (33) may be imposed by adjusting the distance of the traps for fixed $k_{L}$ and $k_{R}$. Besides the line of maximum rectification, Fig. 2 also shows two lines where rectification is zero. At these lines forward and backward fluxes cross. Solving $R=0$ with a Taylor series around $\left(\gamma_{L}, \gamma_{R}\right)=(0,0)$ gives, up to second order in friction coefficients, the two approximate solutions

$$
\begin{aligned}
k_{R}= & k_{L}\left[\frac{m_{2}}{m_{1}} \pm \frac{1}{2 k} \sqrt{\frac{m_{2} \gamma_{L} \gamma_{R}^{3}}{m_{1}^{3}}}\right] \\
& +k\left[\frac{m_{2}}{m_{1}}\left(1 \pm \frac{2 m_{1} m_{2} \gamma_{R}+\left(m_{1}^{2}+m_{2}^{2}\right) \gamma_{L}}{2 \sqrt{m_{1} m_{2}^{3} \gamma_{L} \gamma_{R}}}\right)-1\right] \\
& \pm \frac{1}{2} \sqrt{\frac{m_{2} \gamma_{L} \gamma_{R}^{3}}{m_{1}^{3}}}+\gamma_{R} \frac{\left(m_{1}^{2}+m_{2}^{2}\right) \gamma_{L}+m_{1} m_{2} \gamma_{R}}{2 m_{1}^{2}\left(m_{2}-m_{1}\right)}
\end{aligned}
$$

The term $\pm \frac{1}{2 k} \sqrt{m_{2} \gamma_{L} \gamma_{R}^{3} / m_{1}^{3}}$ in Eq. (35) makes the slopes of the two zero-rectification lines different from each other and also from the maximum-rectification line. This difference is, however, hardly noticeable for weak dissipation as in Fig. 2.

Interestingly, along the maximum line (33) the rectification no longer depends on the spring constants of the model [see Eqs. (21) and (32)],

$$
R= \begin{cases}1-\frac{a+g}{1+a g} & \text { if } a>1, g>1 \text { or } a<1, g<1 \\ 1-\frac{1+a g}{a+g} & \text { if } a>1, g<1 \text { or } a<1, g>1,\end{cases}
$$

it only depends on the mass and friction coefficient ratios $a$ and $g$ :

$$
a=m_{2} / m_{1}, \quad g=\gamma_{R} / \gamma_{L} .
$$

Besides a high value of $R$, it is desirable to have a significant current $J_{\max }$ [17]. Using again Eq. (33) in the expression for the currents [Eq. (21)], the maximum current $J_{\max }=$ $\max (|J|,|\tilde{J}|)$ is

$$
J_{\max }= \begin{cases}\frac{k_{B} g \gamma_{L} k^{2}\left|T_{L}-T_{R}\right|}{(a+g)\left(g \gamma_{L}^{2}\left(k_{L}+k\right)+k^{2} m_{1}\right)} & \text { if }\left\{\begin{array}{l}
a>1, g>1 \\
\text { or } a<1, g<1,
\end{array}\right. \\
\frac{k_{B} g \gamma_{L} k^{2}\left|T_{L}-T_{R}\right|}{(1+a g)\left(g \gamma_{L}^{2}\left(k_{L}+k\right)+k^{2} m_{1}\right)} & \text { if }\left\{\begin{array}{l}
a>1, g<1 \\
\text { or } a<1, g>1 .
\end{array}\right.\end{cases}
$$

Now we analyze how the parameters $a$ and $g$ affect the maximum current $J_{\max }$ in Eqs. (38). To do this, we can divide the $a g$ plane in four quadrants by the axes $a=1$ and $g=1$ (in those axes $R=0$ ). In Eqs. (38) the parameter $a$ appears only in the denominator; thus for a higher $a$, a smaller current is found. The quadrants with $a<1$ will be better for achieving large currents. $g$ appears both in the numerator and denominator so there is no obvious advantageous quadrant for this parameter.

Equations (36) are symmetric upon the transformations $a \leftrightarrow 1 / a$ and $g \leftrightarrow 1 / g$. Using a logarithmic scale for $a$ and $g$, the resulting $R$ map is symmetric with respect to the $a=1$ and $g=1$ axes. We can thus limit ourselves to analyze the quadrant $a>1, g>1$.

Figure 3 shows the rectification given by Eq. (36) in terms of $a$ and $g$. Along any diagonal line (parallel to the solid cyan or the dashed green lines), the maximum value is at the center, that is, when $a=g$. For constant $a$, a larger $g$ always increases $R$, but making the ratio between friction coefficients $g$ arbitrarily large is not realistic in a trapped-ion setup. Making $\gamma_{L}$ go to zero decouples one of the ions from the bath, so the heat current tends to vanish in any direction. Also, increasing $\gamma_{R}$ arbitrarily is impossible since it is a function of the laser detuning [Eqs. (31)], which is physically bounded by the existence of other levels. Although Eqs. (31) suggest that boosting the laser intensity can also increase the friction coefficient, this is not an option since Eqs. (31) are just an approximation for low laser intensities. When going to higher intensities, the emission or absorption of photons by the ion is saturated and the friction coefficient reaches a finite value proportional to the width $\Gamma$ of the excited state [31]. As a compromise between feasibility and high $R$, let as assume the ratio between the friction coefficients $g$ to be equal to the mass ratio $a$. As shown in Fig. 3, along the solid cyan 


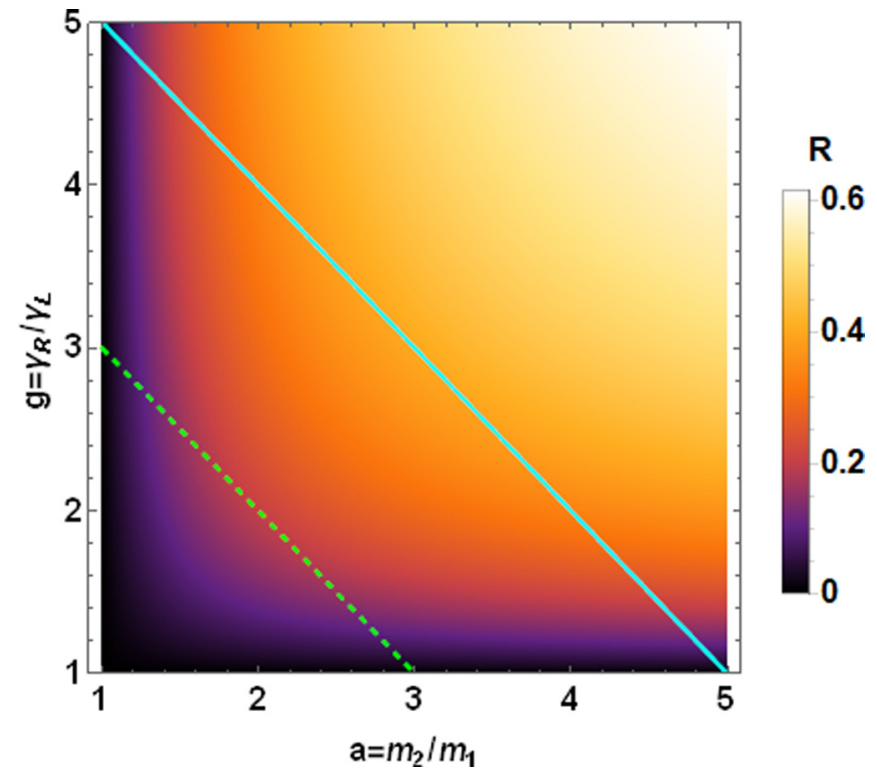

FIG. 3. Rectification factor, $R$, given by Eqs. (36).

and dashed green diagonal lines the maximum $R$ is achieved for $a=g$. The effect of varying the common value $c$ of $a$ and $g, c=a=g$, may be seen in Fig. 4, which shows the rectification in Eq. (36). $R$ tends to 1 for large $c$.

\section{B. Spectral match-mismatch approach to rectification}

If there is a good match between the phonon spectra of the ions (i.e., their peaks overlap in a broad range of frequencies) for a certain baths configuration, and mismatch when the baths exchange, the system will present heat rectification [2,3]. We have studied the spectra of the ions in our model for several sets of parameters exhibiting no rectification or strong rectification. The spectra are calculated through the spectral density matrix. For a realvalued stochastic process $\vec{x}(t)$, its spectral density matrix is defined as [24]

$$
\mathbb{S}_{\vec{x}}(\omega) \equiv\left\langle\vec{X}(\omega) \vec{X}^{\top}(-\omega)\right\rangle,
$$

with $\vec{X}(\omega)$ being the Fourier transform of $\vec{x}(t)$ (we use the convention of factors of 1 and $1 /(2 \pi)$ for the transform and the inverse transform). A justification of the use of the spectral density matrix to understand heat transport arises from the Wiener-Khinchin theorem [24], which says that the correlation matrix of a stationary stochastic process in the steady state is the inverse Fourier transform of its spectral density matrix, $\left\langle\vec{r}(t) \vec{r}^{\top}(t+\tau)\right\rangle=\mathcal{F}^{-1}\left[\mathbb{S}_{\vec{r}}(\omega)\right](\tau)$. Thus, the covariance matrix in the steady state is

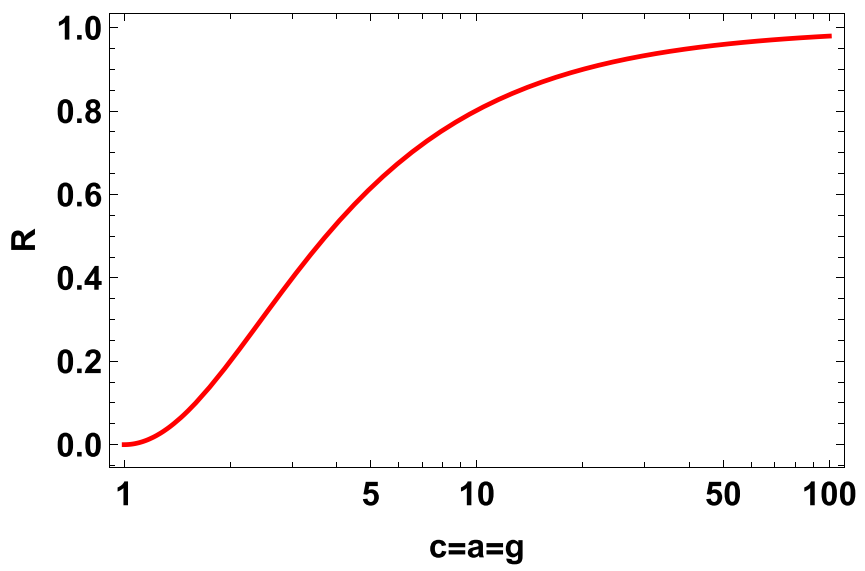

FIG. 4. Rectification for different values of $c=m_{2} / m_{1}=\gamma_{R} / \gamma_{L}$ when the maximum condition in the $k_{L} k_{R}$ plane is satisfied [Eq. (33)].

$$
\mathbb{C}^{\text {s.s. }}=\frac{1}{2 \pi} \int_{-\infty}^{\infty} d \omega \mathbb{S}_{\vec{r}}(\omega) .
$$

Equation (40) directly connects the spectral density matrix to the steady-state temperature since $T_{1}^{\text {s.s. }}=m_{1} C_{3,3}^{\text {s.s. }} / k_{B}$ and $T_{2}^{\text {s.s. }}=m_{2} C_{4,4}^{\text {s.s. }} / k_{B}$, and, therefore, to the heat currents [see Eqs. (9) and (20)].

The Fourier transform of the vector process $\vec{r}(t)$ describing the evolution of our system [see Eq. (5)] is $\vec{R}(\omega)=$ $(i \omega-\mathbb{A})^{-1} \mathbb{L} \vec{\Xi}(\omega)$ with $\vec{\Xi}(\omega)$ being the Fourier transform of the white noise $\vec{\xi}(t)$. Note that $\vec{\Xi}(\omega)$ is not square integrable; however, its spectral density is $\mathbb{S}_{\vec{\xi}}(\omega)=2 \mathbb{D}$ [24], which is flat as expected for a white noise. Therefore, the spectral density matrix of the system is

$$
\mathbb{S}_{\vec{r}}(\omega)=2(\mathbb{A}-i \omega)^{-1} \mathbb{L} \mathbb{D L}^{\top}(\mathbb{A}+i \omega)^{-\top} .
$$

The imaginary part of the eigenvalues of the dynamical matrix $\mathbb{A}$ correspond to the peaks in the spectrum whereas the real part dictates their width. Equation (41) gives after direct computation

$$
\mathbb{S}_{\vec{r}}(\omega)=2 k_{B} \frac{\gamma_{L} T_{L} \mathbb{S}_{L}(i \omega)+\gamma_{L} T_{R} \mathbb{S}_{R}(i \omega)}{\left(m_{1} m_{2}\right)^{2} P_{\mathbb{A}}(i \omega) P_{\mathbb{A}}(-i \omega)},
$$

where $P_{\mathbb{A}}(\lambda)$ is the characteristic polynomial of the dynamical matrix $\mathbb{A}$ and $\mathbb{S}_{L}(\omega), \mathbb{S}_{R}(\omega)$ are the matrix polynomials in the angular frequency $\omega$ whose coefficients are defined in Appendix B. We give the spectral densities for the velocities, $\mathcal{S}_{1} \equiv \mathbb{S}_{3,3}(\omega)=\left\langle R_{3}(\omega) R_{3}(-\omega)\right\rangle$ for the left ion and $\mathcal{S}_{2} \equiv \mathbb{S}_{4,4}(\omega)=\left\langle R_{4}(\omega) R_{4}(-\omega)\right\rangle$ for the right ion, since they are the elements related to the calculation of the heat current using Eq. (40),

$$
\begin{aligned}
& \mathcal{S}_{1}(\omega)=2 k_{B} \frac{\gamma_{R} k^{2} T_{R} \omega^{2}+\gamma_{L} T_{L}\left[\omega^{4}\left(\gamma_{R}^{2}-2 k m_{2}-2 k_{R} m_{2}\right)+\omega^{2}\left(k+k_{R}\right)^{2}+m_{2}^{2} \omega^{6}\right]}{\left(m_{1} m_{2}\right)^{2} P_{\mathbb{A}}(i \omega) P_{\mathbb{A}}(-i \omega)}, \\
& \mathcal{S}_{2}(\omega)=2 k_{B} \frac{\gamma_{L} k^{2} T_{L} \omega^{2}+\gamma_{R} T_{R}\left[\omega^{4}\left(\gamma_{L}^{2}-2 k m_{1}-2 k_{L} m_{1}\right)+\omega^{2}\left(k+k_{L}\right)^{2}+m_{1}^{2} \omega^{6}\right]}{\left(m_{1} m_{2}\right)^{2} P_{\mathbb{A}}(i \omega) P_{\mathbb{A}}(-i \omega)} .
\end{aligned}
$$



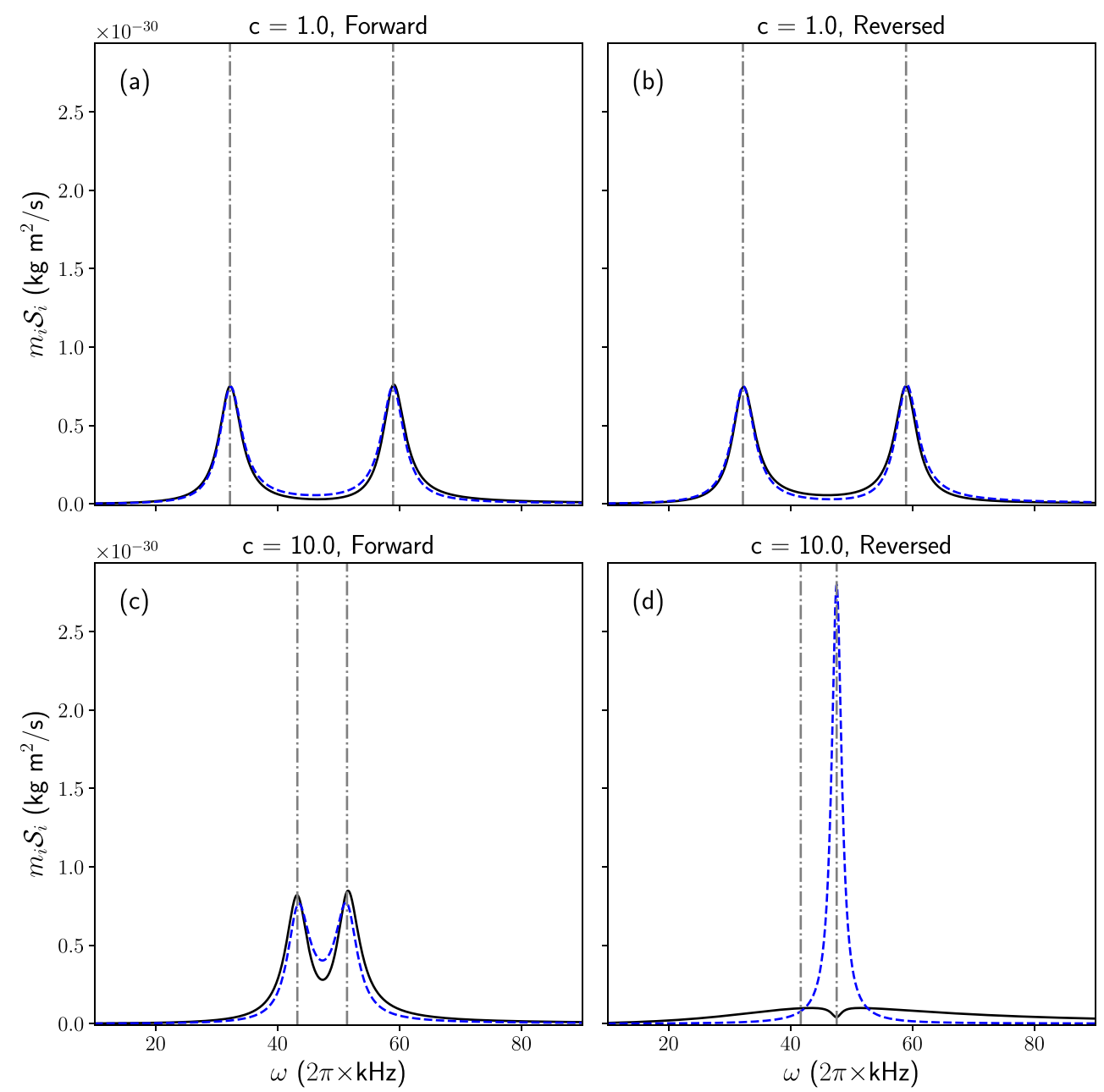

FIG. 5. Spectral densities of the velocities of the ions $\left(r_{3}\right.$ and $\left.r_{4}\right)$ corresponding to $T_{L}=\tilde{T}_{R}=2 \mathrm{mK}, T_{R}=\tilde{T}_{L}=1 \mathrm{mK}$, and two values of $c$ in Fig. 4: [(a),(b)] $c=1$ and $[(\mathrm{c}),(\mathrm{d})] c=10$. Solid black lines are for the left ion spectral density $\mathcal{S}_{1}(\omega)$ and dashed blue lines for the right ion spectral density $\mathcal{S}_{2}(\omega)$. Dot-dashed vertical lines mark the frequencies of the normal modes of the system. The spectra are multiplied by their corresponding masses so that the areas are proportional to the steady-state temperatures [see Eq. (40)]. (a) and (b) correspond to $R=0$ : the overlap between the phonon bands is the same in forward and reversed configurations. (c) and (d) correspond to $R \approx 0.8$ : in (c) the forward configuration, the phonons match better than in (d) the reversed configuration.

Figure 5 depicts a series of plots of the spectra given by Eq. (43), corresponding to two points in Fig. 4. (The calculation for the reverse bias is done with the substitutions in Table I.) For $c=1$ [Figs. 5(a) and 5(b)] there is no rectification, since the spectra match in the forward [Fig. 5(a)] and reversed [Fig. 5(b)] configurations. However, for $c=10$ [Figs. $5(\mathrm{c})$ and $5(\mathrm{~d}), R \approx 0.8$ ] the picture is very different: there is a good match between the spectra in the forward configuration but not for the reversed configuration. It is interesting to analyze how the system changes from $c=1$ to $c=10$ using the dissipative normal modes of the system, which may be found by diagonalizing the dynamical matrix $\mathbb{A}$ [Eq. (6)]. The frequencies of the peaks in Fig. 5 are given by the imaginary part of the eigenvalues of $\mathbb{A}$. Likewise, the width depends on the real part of the eigenvalues. For the forward configuration, the normal frequencies (position of the peaks) come closer to each other as $c$ is increased, while the widths remain practically constant. To understand why the real part remains practically constant, we recall that we have chosen to work with spring constants that satisfy Eq. (33) and making the mass and friction coefficient ratios equal to $c$, i.e., $c \equiv m_{2} / m_{1}=\gamma_{R} / \gamma_{L}$. The (dissipative) terms in $\mathbb{A}$ responsible for the real parts in the eigenvalues are, for the forward configuration, $\gamma_{L} / m_{1}$ and $\gamma_{R} / m_{2}=\left(c \gamma_{L}\right) /\left(c m_{1}\right)=\gamma_{L} / m_{1}$, which are constant for every value of $c$. On the contrary, in the reverse bias configuration the dissipative terms in the dynamical matrix are $\tilde{\gamma}_{L} / m_{1}=$ $\gamma_{R} / m_{1}=c \gamma_{L} / m_{1}$ and $\tilde{\gamma}_{R} / m_{2}=\gamma_{L} /\left(c m_{1}\right)$, with opposite behavior with respect to $c$. The real parts of the eigenvalues also behave quite differently for reverse bias: one of them gets closer to the imaginary axis for $c=10$ [see Fig. 5(d)], where this mode concerns mostly the right ion, the only one excited at the peak frequency, while the other eigenvalue moves far from the imaginary axis so a peak is not noticeable at the imaginary value (left dot-dashed line) anymore. 


\section{CONCLUSIONS}

We have studied heat rectification in a model composed of two coupled harmonic oscillators connected to Langevin baths, which could be realized with trapped ions and optical molasses. This simple model allows analytical treatment but still has enough complexity to examine different ingredients that can produce rectification. Our results demonstrate in a simple but realistic model that harmonic systems can rectify heat current if they have features which depend on the temperature [16]. We implement this notion of temperaturedependent features by defining the baths exchange operation as an exchange of both temperatures and coupling parameters of the baths to the system. The temperature dependence of the bath-system coupling occurs naturally in laser-cooled trapped-ion setups.

We have also studied the phonon spectra of the system, aided by a normal mode analysis, comparing the match and mismatch of the phonon bands, to reach the conclusion that the band match-mismatch description for heat rectification is also valid for systems which are purely harmonic, as long as there are temperature-dependent features. We hope this article sheds more light on the topic of heat rectification and that encourages more research regarding its physical implementation on chains of trapped ions.

\section{ACKNOWLEDGMENTS}

We thank Daniel Alonso for fruitful discussions and comments. This work was supported by the Basque Country Government (Grant No. IT986-16), by Grants No. PGC2018-101355-B-I00 (MCIU/AEI/FEDER,UE) and No. FIS2016-80681P, and by the Spanish MICINN and European Union (FEDER) (Grant No. FIS2017-82855-P). M.A.S. acknowledges support by the Basque Government predoctoral program (Grant No. PRE-2019-2-0234).

\section{APPENDIX A: FULL SET OF STEADY-STATE EQUATIONS FOR THE COMPONENTS OF $\mathbb{C}^{\text {s.s. }}$}

Here is the full set of equations for the covariance matrix elements in the steady state. We use the shorthand notation $\langle\cdots\rangle=$ $\langle\cdots\rangle^{\text {s.s. }}$; i.e., all averages are in the steady state:

$$
\begin{aligned}
& \frac{2 k\left\langle p_{2} q_{1}\right\rangle}{m_{1} m_{2}}+\frac{2 \gamma_{L}\left\langle p_{1}^{2}\right\rangle}{m_{1}^{3}}=\frac{2 D_{L}}{m_{1}^{2}}, \\
& -\frac{2 k\left\langle p_{2} q_{1}\right\rangle}{m_{2}^{2}}+\frac{2 \gamma_{R}\left\langle p_{2}^{2}\right\rangle}{m_{2}^{3}}=\frac{2 D_{R}}{m_{2}^{2}}, \\
& -\frac{\left(k_{L}+k\right)\left\langle q_{1} q_{2}\right\rangle}{m_{1}}+\frac{k\left\langle q_{2}^{2}\right\rangle}{m_{1}}+\frac{\gamma_{L}\left\langle p_{2} q_{1}\right\rangle}{m_{1} m_{2}}+\frac{\left\langle p_{1} p_{2}\right\rangle}{m_{1} m_{2}}=0, \\
& \frac{\left(k_{L}+k\right)\left\langle p_{2} q_{1}\right\rangle}{m_{1} m_{2}}-\frac{\left(k_{R}+k\right)\left\langle p_{2} q_{1}\right\rangle}{m_{2}^{2}}+\frac{\gamma_{L}\left\langle p_{1} p_{2}\right\rangle}{m_{1}^{2} m_{2}}+\frac{\gamma_{R}\left\langle p_{1} p_{2}\right\rangle}{m_{1} m_{2}^{2}}=0, \\
& -\frac{\left(k_{L}+k\right)\left\langle q_{1}^{2}\right\rangle}{m_{1}}+\frac{k\left\langle q_{1} q_{2}\right\rangle}{m_{1}}+\frac{\left\langle p_{1}^{2}\right\rangle}{m_{1}^{2}}=0, \\
& -\frac{\left(k_{R}+k\right)\left\langle q_{2}^{2}\right\rangle}{m_{2}}+\frac{k\left\langle q_{1} q_{2}\right\rangle}{m_{2}}+\frac{\left\langle p_{2}^{2}\right\rangle}{m_{2}^{2}}=0, \\
& -\frac{\left(k_{R}+k\right)\left\langle q_{1} q_{2}\right\rangle}{m_{2}}+\frac{k\left\langle q_{1}^{2}\right\rangle}{m_{2}}-\frac{\gamma_{R}\left\langle p_{2} q_{1}\right\rangle}{m_{2}^{2}}+\frac{\left\langle p_{1} p_{2}\right\rangle}{m_{1} m_{2}}=0 .
\end{aligned}
$$

\section{APPENDIX B: COMPLETE EXPRESSIONS FOR THE SPECTRAL DENSITY MATRIX}

In Sec. VI we used the characteristic polynomial $P_{\mathbb{A}}(\lambda)$ of the dynamical matrix $\mathbb{A}$ for the calculation of the spectral density matrix. $P_{\mathbb{A}}(\lambda)$ is defined as

$$
\begin{aligned}
P_{\mathbb{A}}(\lambda) \equiv & \operatorname{det}(\mathbb{A}-\lambda) \\
= & \lambda^{4}+\lambda^{3}\left(\frac{\gamma_{L}}{m_{1}}+\frac{\gamma_{R}}{m_{2}}\right)+\lambda^{2} \frac{\left(\gamma_{L} \gamma_{R}+m_{2}\left(k+k_{L}\right)+m_{1}\left(k+k_{R}\right)\right)}{m_{1} m_{2}}+\lambda \frac{\left(\gamma_{R}\left(k+k_{L}\right)+\gamma_{L}\left(k+k_{R}\right)\right)}{m_{1} m_{2}} \\
& +\frac{k\left(k_{L}+k_{R}\right)+k_{L} k_{R}}{m_{1} m_{2}} .
\end{aligned}
$$


We also used the polynomials $\mathbb{S}_{L}(\lambda)=\sum_{n=0}^{6} \lambda^{n} \mathbb{S}_{L, n}$ and $\mathbb{S}_{R}(\lambda)=\sum_{n=0}^{6} \lambda^{n} \mathbb{S}_{R, n}$. There are 14 different polynomial coefficients, which are $4 \times 4$ matrices. This is the full list of coefficients:

$$
\begin{aligned}
& \mathbb{S}_{L, 0}=\left(\begin{array}{cccc}
\left(k+k_{R}\right)^{2} & k\left(k+k_{R}\right) & 0 & 0 \\
k\left(k+k_{R}\right) & k^{2} & 0 & 0 \\
0 & 0 & 0 & 0 \\
0 & 0 & 0 & 0
\end{array}\right), \\
& \mathbb{S}_{R, 0}=\left(\begin{array}{cccc}
k^{2} & k\left(k+k_{L}\right) & 0 & 0 \\
k\left(k+k_{L}\right) & \left(k+k_{L}\right)^{2} & 0 & 0 \\
0 & 0 & 0 & 0 \\
0 & 0 & 0 & 0
\end{array}\right) \\
& \mathbb{S}_{L, 1}=\left(\begin{array}{cccc}
0 & k \gamma_{R} & -\left(k+k_{R}\right)^{2} & -k\left(k+k_{R}\right) \\
-k \gamma_{R} & 0 & -k\left(k+k_{R}\right) & -k^{2} \\
\left(k+k_{R}\right)^{2} & k\left(k+k_{R}\right) & 0 & 0 \\
k\left(k+k_{R}\right) & k^{2} & 0 & 0
\end{array}\right), \\
& \mathbb{S}_{R, 1}=\left(\begin{array}{cccc}
0 & -k \gamma_{L} & -k^{2} & -k\left(k+k_{L}\right) \\
k \gamma_{L} & 0 & -k\left(k+k_{L}\right) & -\left(k+k_{L}\right)^{2} \\
k^{2} & k\left(k+k_{L}\right) & 0 & 0 \\
k\left(k+k_{L}\right) & \left(k+k_{L}\right)^{2} & 0 & 0
\end{array}\right), \\
& \mathbb{S}_{L, 2}=\left(\begin{array}{cccc}
2\left(k+k_{R}\right) m_{2}-\gamma_{R}^{2} & k m_{2} & 0 & -k \gamma_{R} \\
k m_{2} & 0 & k \gamma_{R} & 0 \\
0 & k \gamma_{R} & -\left(k+k_{R}\right)^{2} & -k\left(k+k_{R}\right) \\
-k \gamma_{R} & 0 & -k\left(k+k_{R}\right) & -k^{2}
\end{array}\right) \text {, } \\
& \mathbb{S}_{R, 2}=\left(\begin{array}{cccc}
0 & k m_{1} & 0 & k \gamma_{L} \\
k m_{1} & 2\left(k+k_{L}\right) m_{1}-\gamma_{L}^{2} & -k \gamma_{L} & 0 \\
0 & -k \gamma_{L} & -k^{2} & -k\left(k+k_{L}\right) \\
k \gamma_{L} & 0 & -k\left(k+k_{L}\right) & -\left(k+k_{L}\right)^{2}
\end{array}\right), \\
& \mathbb{S}_{L, 3}=\left(\begin{array}{cccc}
0 & 0 & \gamma_{R}^{2}-2\left(k+k_{R}\right) m_{2} & -k m_{2} \\
0 & 0 & -k m_{2} & 0 \\
2\left(k+k_{R}\right) m_{2}-\gamma_{R}^{2} & k m_{2} & 0 & -k \gamma_{R} \\
k m_{2} & 0 & k \gamma_{R} & 0
\end{array}\right) \\
& \mathbb{S}_{R, 3}=\left(\begin{array}{cccc}
0 & 0 & 0 & -k m_{1} \\
0 & 0 & -k m_{1} & \gamma_{L}^{2}-2\left(k+k_{L}\right) m_{1} \\
0 & k m_{1} & 0 & k \gamma_{L} \\
k m_{1} & 2\left(k+k_{L}\right) m_{1}-\gamma_{L}^{2} & -k \gamma_{L} & 0
\end{array}\right), \\
& \mathbb{S}_{L, 4}=\left(\begin{array}{cccc}
m_{2}^{2} & 0 & 0 & 0 \\
0 & 0 & 0 & 0 \\
0 & 0 & \gamma_{R}^{2}-2\left(k+k_{R}\right) m_{2} & -k m_{2} \\
0 & 0 & -k m_{2} & 0
\end{array}\right), \quad \mathbb{S}_{R, 4}=\left(\begin{array}{cccc}
0 & 0 & 0 & 0 \\
0 & m_{1}^{2} & 0 & 0 \\
0 & 0 & 0 & -k m_{1} \\
0 & 0 & -k m_{1} & \gamma_{L}^{2}-2\left(k+k_{L}\right) m_{1}
\end{array}\right), \\
& \mathbb{S}_{L, 5}=\left(\begin{array}{cccc}
0 & 0 & -m_{2}^{2} & 0 \\
0 & 0 & 0 & 0 \\
m_{2}^{2} & 0 & 0 & 0 \\
0 & 0 & 0 & 0
\end{array}\right), \quad \mathbb{S}_{R, 5}=\left(\begin{array}{cccc}
0 & 0 & 0 & 0 \\
0 & 0 & 0 & -m_{1}^{2} \\
0 & 0 & 0 & 0 \\
0 & m_{1}^{2} & 0 & 0
\end{array}\right),
\end{aligned}
$$




$$
\mathbb{S}_{L, 6}=\left(\begin{array}{cccc}
0 & 0 & 0 & 0 \\
0 & 0 & 0 & 0 \\
0 & 0 & -m_{2}^{2} & 0 \\
0 & 0 & 0 & 0
\end{array}\right), \quad \mathbb{S}_{R, 6}=\left(\begin{array}{cccc}
0 & 0 & 0 & 0 \\
0 & 0 & 0 & 0 \\
0 & 0 & 0 & 0 \\
0 & 0 & 0 & -m_{1}^{2}
\end{array}\right)
$$

[1] C. Starr, Physics 7, 15 (1936).

[2] M. Terraneo, M. Peyrard, and G. Casati, Phys. Rev. Lett. 88, 094302 (2002).

[3] B. Li, L. Wang, and G. Casati, Phys. Rev. Lett. 93, 184301 (2004).

[4] E. Pereira, Europhys. Lett. 126, 14001 (2019).

[5] N. Roberts and D. Walker, Int. J. Therm. Sci. 50, 648 (2011).

[6] N. Li, J. Ren, L. Wang, G. Zhang, P. Hänggi, and B. Li, Rev. Mod. Phys. 84, 1045 (2012).

[7] T. Ma and Y. Wang, in Carbon Based Nanomaterials for Advanced Thermal and Electrochemical Energy Storage and Conversion, Micro and Nano Technologies, edited by R. Paul, V. Etacheri, Y. Wang, and C.-T. Lin (Elsevier, Amsterdam, 2019), pp. 103-119.

[8] A. Riera-Campeny, M. Mehboudi, M. Pons, and A. Sanpera, Phys. Rev. E 99, 032126 (2019).

[9] N. Li, P. Hänggi, and B. Li, Europhys. Lett. 84, 40009 (2008).

[10] B. Hu, L. Yang, and Y. Zhang, Phys. Rev. Lett. 97, 124302 (2006).

[11] N. Zeng and J.-S. Wang, Phys. Rev. B 78, 024305 (2008).

[12] D. Segal and A. Nitzan, Phys. Rev. Lett. 94, 034301 (2005).

[13] D. Segal and A. Nitzan, J. Chem. Phys. 122, 194704 (2005).

[14] G. Katz and R. Kosloff, Entropy 18, 186 (2016).

[15] G. Benenti, G. Casati, C. Mejía-Monasterio, and M. Peyrard, in Thermal Transport in Low Dimensions: From Statistical Physics to Nanoscale Heat Transfer, edited by S. Lepri (Springer, Cham, 2016), pp. 365-407.

[16] E. Pereira, Phys. Rev. E 96, 012114 (2017).

[17] M. A. Simón, S. Martínez-Garaot, M. Pons, and J. G. Muga, Phys. Rev. E 100, 032109 (2019).
[18] N. Yang, N. Li, L. Wang, and B. Li, Phys. Rev. B 76, 020301(R) (2007).

[19] E. Pereira and R. R. Ávila, Phys. Rev. E 88, 032139 (2013).

[20] S. Chen, E. Pereira, and G. Casati, Europhys. Lett. 111, 30004 (2015).

[21] C. D. Bruzewicz, J. Chiaverini, R. McConnell, and J. M. Sage, Appl. Phys. Rev. 6, 021314 (2019).

[22] M. Pons, Y. Y. Cui, A. Ruschhaupt, M. A. Simón, and J. G. Muga, Europhys. Lett. 119, 64001 (2017).

[23] T. J. Alexander, Phys. Rev. E 101, 062122 (2020).

[24] S. Särkkä and A. Solin, Applied Stochastic Differential Equations (Cambridge University Press, Cambridge, UK, 2019), pp. 92-93.

[25] Z. Rieder, J. L. Lebowitz, and E. Lieb, J. Math. Phys. 8, 1073 (1967).

[26] A. Casher and J. L. Lebowitz, J. Math. Phys. 12, 1701 (1971).

[27] D. Leibfried, R. Blatt, C. Monroe, and D. Wineland, Rev. Mod. Phys. 75, 281 (2003).

[28] S. Chu, L. Hollberg, J. E. Bjorkholm, A. Cable, and A. Ashkin, Phys. Rev. Lett. 55, 48 (1985).

[29] C. Cohen-Tannoudji, in Fundamental Systems in Quantum Optics, edited by J. Dalibard, J. M. Raimond, and J. Zinn-Justin (North-Holland, Amsterdam, 1993).

[30] H. Metcalf and P. Van der Straten, Laser Cooling and Trapping, Graduate Texts in Contemporary Physics (Springer-Verlag, New York, 1999).

[31] H. J. Metcalf and P. van der Straten, J. Opt. Soc. Am. B 20, 887 (2003).

[32] A. Ruiz, D. Alonso, M. B. Plenio, and A. del Campo, Phys. Rev. B 89, 214305 (2014). 